\title{
The impact of completeness of last transurethral resection of bladder tumors on the outcomes of radical cystectomy
}

\author{
Stefania Zamboni ${ }^{1,3} \cdot$ Marco Moschini $^{1,2} \cdot$ Andrea Gallina $^{2} \cdot$ Renzo Colombo $^{2} \cdot$ Francesco Montorsi $^{2}$. \\ Alberto Briganti $^{2} \cdot$ Andrea Salonia $^{2} \cdot$ Alessandro Antonelli $^{3} \cdot$ Claudio Simeone $^{3} \cdot$ Sandra Belotti $^{3} \cdot$ Luca Cristinelli $^{3}$. \\ Agostino Mattei $^{1} \cdot$ Philipp Baumeister ${ }^{1}$
}

Received: 28 November 2018 / Accepted: 15 March 2019

(c) Springer-Verlag GmbH Germany, part of Springer Nature 2019

\begin{abstract}
Purpose To evaluate the role of a complete transurethral resection of bladder tumors (c-TURBT) on oncological outcomes after radical cystectomy (RC) and its relationship with adverse pathological features.

Methods We retrospectively analyzed data of 727 patients treated with RC and bilateral pelvic lymph node dissection at three tertiary referral centers. Possible c-TURBT was reported by the treating surgeon. Multivariable Cox regression analyses were used to assess the relationship of c-TURBT and survival outcomes after surgery in 1:1 propensity score-matched cohort adjusted for age and gender. Moreover, multivariable logistic regression (MVA) was built to predict the relationship between c-TURBT and pT3-T4 stages at RC, lymph node invasion (LNI) and positive soft tissue surgical margin (STSM). Results A total of $433(60 \%)$ patients received a c-TURBT. 3.0\% of patients with a c-TURBT achieved a pT0-pTa-pTis status vs. $2.0 \%$ of patients with incomplete TURBT. At multivariable Cox regression analyses, c-TURBT was not associated with survival outcomes. At MVA, incompleteness of TURBT was significantly associated with a pT3-T4 stage [odds ratio (OR) $8.04,95 \%$ confidence interval (CI) 2.33-27.67, $p=0.001]$. No significant association was found between c-TURBT, LNI and STSM.

Conclusion We found a low rate of achievement of pT0 stage at RC. An incomplete TURBT before RC represented a predictor of pT3-T4 stages, but no effect of a c-TURBT was shown on survival outcomes. Given the current inadequacy of clinical staging strategies with more than $50 \%$ of extravesical disease being under-staged, our results could improve patients selection for NAC, driving the decision-making in doubtful cases.
\end{abstract}

Keywords Bladder cancer $\cdot$ Radical cystectomy $\cdot$ Transurethral resection $\cdot$ TURBT $\cdot$ Incomplete

\section{Introduction}

Bladder cancer (BCa) is the second most common genitourinary malignancy with 81,190 estimated new diagnosis in the 2018 in the USA only [1]. Transurethral resection of bladder tumors (TURBT) represents an important diagnostic tool in

Stefania Zamboni

stefania.zamboni@libero.it

1 Klinik für Urologie, Luzerner Kantonsspital, Lucerne, Switzerland

2 Unit of Urology, Division of Oncology, IRCCS Ospedale San Raffaele, URI Milan, Milan, Italy

3 Urology Unit, ASST Spedali Civili, Department of Medical and Surgical Specialties, Radiological Science and Public Health, University of Brescia, Brescia, Italy the correct tumor staging and the gold standard treatment for non-muscle-invasive $\mathrm{BCa}$ (with intravesical chemo- or immunotherapy) [2]. The role of completeness of TURBT in non-muscle-invasive BCa was largely evaluated, and several studies reported a benefit in recurrence and survival outcomes conferred by a maximal extended TURBT before bladder instillation [3-5].

However, scarce data exist regarding the opportunity to complete a TURBT in patients affected by macroscopic invasive disease at endoscopic evaluation. According to the risk of bladder perforation and subsequent tumor spreading, the intraoperative evaluation sometimes leads to the decision to perform an incomplete TURBT, especially when tumors are extended, deep or located in particular areas (such as diverticula). Sparse data exist regarding the potential impact of the incompleteness of resection before radical cystectomy 
(RC) and its role on recurrence and survival outcomes in locally advanced disease. We therefore evaluated a cohort of patients treated with $\mathrm{RC}$ for clinical non-metastatic $\mathrm{BCa}$ in three tertiary referral centers, stratifying according to the completeness of TURBT before RC.

\section{Materials and methods}

We retrospectively investigated 1089 patients who underwent RC and pelvic lymph node dissection (PLND) between 2001 and 2018 at three tertiary referral centers.

$\mathrm{RC}$ was suggested according to EAU guidelines [6] in case of very high risk non-muscle-invasive $\mathrm{BCa}$ or in case of confined muscle-invasive bladder cancer with curative intent or in case of non-confined disease with palliative intent. This study included 727 patients with localized disease treated with RC and PLND with curative intent, whereas 362 patients treated with RC with a palliative intent or with neoadjuvant chemotherapy (NAC) were excluded. All included cases underwent diagnostic TURBT with tumor removal prior to radical cystectomy. We considered, for all the analyses, the last TURBT before radical cystectomy which includes the second TURBT, in case of patients who underwent repeated TURBT. Re-TURBT was indicated and performed according to guidelines [2]. This last resection was reported by the treating surgeon as complete or incomplete. Patients were staged preoperatively with pelvic/abdominal computerized tomography (CT), bone scan when indicated and chest X-ray or thoracic CT scan. Dedicated pathologists examined all TURBT and RC specimens. Tumor grade was evaluated according to $1973 \mathrm{WHO}$ grading system [7] for all patients who underwent TURBT or RC between 2001 and 2004 and, for patients submitted to surgery later, according to grading WHO 2004 [8].

Concomitant carcinoma in situ (CIS) was defined as the presence of CIS at TURBT or RC specimens' in association with another pathological stage; the term lymphovascular invasion (LVI) indicated the presence of tumor cells within an endothelium-lined space without underlying muscular walls [9]. We considered as variant histology any presence of urothelial or nonurothelial variants at the pathological report [10]. Number of tumors and complete resection were visually evaluated, and monofocality was defined as the presence of a single papillary disease whereas complete resection as the absence of macroscopic residual tumor at the end of the TURBT. Pathologic data of RC included grade, local and lymph nodal staging (according to VI edition TNM classification) [11], LVI [9], concomitant CIS and soft tissue surgical margin (STSM) status defined as any surgical positive margins and excluded the ureteral and urethral ones. Recurrences were defined as appearance of metastasis and/or local recurrences. Adjuvant cisplatin-based chemotherapy was offered to patients with a pT3-4 and/or pN + non-metastatic disease, when patients were fitted and if no neoadjuvant chemotherapy was administered.

Clinical and radiological follow-up consisted of a baseline visit at 3-4 months after surgery. Subsequently, the minimum follow-up consisted of at least two annual visits. Examinations included radiological imaging with CT scan in all patients. In addition to physical examination with laboratory testing, intravenous pyelography, neo-cystoscopy, urine cytology, urethral washings and bone scan were carried out if indicated.

\section{Primary and secondary end points}

Primary endpoint of our study was to assess relationship between c-TURBT and recurrence, cancer-specific mortality (CSM) and overall mortality (OM). The secondary endpoint was to evaluate the relationship between the completeness of TURBT (c-TURBT) and the occurrence of adverse pathologic features, which include pT3-T4 stages, positive STSM or lymph node invasion (LNI) at RC specimen.

\section{Statistical analyses}

Categorical variables were reported as frequencies and proportions, continuous as mean and standard deviation (SD) or median and interquartile ranges 25-75 (IQR). The Kruskal-Wallis and Chi-square tests were used to compare continuous and discrete variables. A 1:1 propensity score matching (PSM) analysis was used to adjust for age and gender. Multivariable Cox regression analyses were used to assess the impact of c-TURBT on recurrence, CSM and OM of the PSM cohort. The Kaplan-Meier method was used to compare survival between groups. The analyses were adjusted for intra- and post-cystectomy features included age, incompleteness of TURBT, pT stage, presence of CIS at $\mathrm{RC}$ specimen, presence of LVI at RC, presence of variant histology at RC, pN status (pN0-pN+), number of lymph nodes removed, positive STSM and administration of adjuvant chemotherapy. Multivariable logistic regression models were used to assess the relationship between c-TURBT on adverse pathological features of the PSM cohort, adjusting analyses for the following precystectomy features: $\mathrm{cT}$ stage (cT3-4 vs. cT1-T2), cN stage (cN0 vs. cN+), hydronephrosis, multifocal disease, CIS and LVI at TURBT (yes vs. no), variant histology, tumor dimension (diameter $\geq$ $3 \mathrm{~cm}$ vs $<3 \mathrm{~cm}$ ). Statistical significance was considered at $p<0.05$. Statistical analyses were performed using STATA $14.0^{\circledR}$ (Stata Corp., College Station, TX, USA). 


\section{Results}

\section{Baseline characteristics}

Patients' characteristics are reported in Table 1. Of the 727 patients included in our study, $433(60 \%)$ received a c-TURBT. In the whole cohort, median age was 69 years (IQR 63-75). The lack of achievement of c-TURBT was related to hydronephrosis $(28 \%$ vs. $17 \%, p<0.001)$ and concomitant CIS (16\% vs. $8.0 \%, p=0.002)$. In 251 patients, the type of TURBT was available and $120(48 \%)$ and $131(52 \%)$ were re-TURBT and diagnostic, respectively. About $31 \%$ of the re-TURBT were reported as incomplete whereas $69 \%$ as complete resection. pT0-pTa or CIS stage achievement occurred in $3.0 \%$ of patients with c-TURBT and in $2.0 \%$ of patients with incomplete TURBT $(<0.001)$.
Table 1 Descriptive characteristics of patients with clinical nonmetastatic bladder cancer (BCa) treated with radical cystectomy (RC) and pelvic lymph node dissection (PLND) between 2001 and 2018 at three tertiary referral centers in whole cohort and propensity scorematched (PSM) cohort

\begin{tabular}{|c|c|c|c|c|c|c|c|c|}
\hline \multirow[t]{2}{*}{ Variables } & \multicolumn{4}{|c|}{ Whole cohort } & \multicolumn{4}{|l|}{ PSM cohort } \\
\hline & $\begin{array}{l}\text { Overall } \\
(N=727, \\
100 \%)\end{array}$ & $\begin{array}{l}\text { c-TURBT } \\
(N=433 \\
60 \%)\end{array}$ & $\begin{array}{l}\text { Incomplete } \\
\text { TURBT }(N=294 \text {, } \\
40 \%)\end{array}$ & $p$ value & $\begin{array}{l}\text { Overall } \\
(N=588, \\
100 \%)\end{array}$ & $\begin{array}{l}\text { c-TURBT } \\
(N=294, \\
50 \%)\end{array}$ & $\begin{array}{l}\text { Incomplete } \\
\text { TURBT }(N=294 \text {, } \\
50 \%)\end{array}$ & $p$ value \\
\hline \multicolumn{9}{|l|}{ Age (years) } \\
\hline Mean & 68 & 68 & 68 & 0.8 & 70 & 68 & 68 & 0.8 \\
\hline Median (IQR) & $69(63-75)$ & $70(63-75)$ & $69(63-75)$ & & $71(67-76)$ & $68(63-75)$ & $68(63-75)$ & \\
\hline \multicolumn{9}{|l|}{ Gender } \\
\hline Male & $614(84 \%)$ & $374(86 \%)$ & $240(82 \%)$ & 0.08 & $475(81 \%)$ & $235(80 \%)$ & $240(82 \%)$ & 0.6 \\
\hline Female & $113(16 \%)$ & $59(14 \%)$ & $54(18 \%)$ & & $113(19 \%)$ & $59(14 \%)$ & $54(18 \%)$ & \\
\hline \multicolumn{9}{|c|}{ Preoperative hydronephrosis } \\
\hline No & $570(79 \%)$ & $359(83 \%)$ & $211(72 \%)$ & $<0.001$ & $453(77 \%)$ & $242(83 \%)$ & $211(72 \%)$ & 0.002 \\
\hline Yes & $156(21 \%)$ & $73(17 \%)$ & $83(28 \%)$ & & $134(23 \%)$ & $51(17 \%)$ & $83(28 \%)$ & \\
\hline \multicolumn{9}{|l|}{ Clinical T stage } \\
\hline $\mathrm{T} 1-\mathrm{T} 2$ & $201(58 \%)$ & $117(62 \%)$ & $84(53 \%)$ & 0.09 & $163(56 \%)$ & $79(60 \%)$ & $84(53 \%)$ & 0.2 \\
\hline $\mathrm{T} 3-\mathrm{T} 4$ & $145(42 \%)$ & $71(38 \%)$ & $74(47 \%)$ & & $126(44 \%)$ & $52(40 \%)$ & $74(47 \%)$ & \\
\hline \multicolumn{9}{|l|}{ TURBT stage $\geq \mathrm{T} 2$} \\
\hline No & $264(37 \%)$ & $270(64 \%)$ & $109(39 \%)$ & 0.5 & $201(35 \%)$ & $92(32 \%)$ & $109(39 \%)$ & 0.06 \\
\hline Yes & $441(63 \%)$ & $155(36 \%)$ & $171(61 \%)$ & & $370(65 \%)$ & $199(68 \%)$ & $171(61 \%)$ & \\
\hline TURBT high grade & $387(93 \%)$ & $268(93 \%)$ & $119(92 \%)$ & 0.8 & $304(92 \%)$ & $185(92.5 \%)$ & $119(92 \%)$ & 0.9 \\
\hline \multicolumn{9}{|l|}{$\begin{array}{l}\text { Concomitant CIS at } \\
\text { TURBT }\end{array}$} \\
\hline No & $623(87 \%)$ & $361(84 \%)$ & $262(92 \%)$ & 0.002 & $515(89 \%)$ & $253(87 \%)$ & $262(92 \%)$ & 0.052 \\
\hline Yes & $92(13 \%)$ & $69(16 \%)$ & $23(8.0 \%)$ & & $61(11 \%)$ & $38(13 \%)$ & $23(8 \%)$ & \\
\hline \multicolumn{9}{|c|}{ Presence of variant histology at TURBT } \\
\hline No & $606(85 \%)$ & $368(86 \%)$ & $232(82 \%)$ & 0.1 & $486(85 \%)$ & $254(88 \%)$ & $232(82 \%)$ & 0.052 \\
\hline Yes & $110(16 \%)$ & $59(14 \%)$ & $50(18 \%)$ & & $86(15 \%)$ & $36(12 \%)$ & $50(18 \%)$ & \\
\hline \multicolumn{9}{|c|}{ Multifocality at TURBT } \\
\hline No & $266(37 \%)$ & $165(39 \%)$ & $101(35 \%)$ & 0.3 & $375(65 \%)$ & $92(32 \%)$ & $109(39 \%)$ & 0.9 \\
\hline Yes & $446(63 \%)$ & $258(61 \%)$ & $188(65 \%)$ & & $202(35 \%)$ & $199(68 \%)$ & $171(61 \%)$ & \\
\hline \multicolumn{9}{|l|}{ Dimension $>3 \mathrm{~cm}$} \\
\hline No & $222(55 \%)$ & $146(59 \%)$ & $76(50 \%)$ & 0.2 & $179(55 \%)$ & $103(67 \%)$ & $129(73 \%)$ & 0.2 \\
\hline Yes & $180(55 \%)$ & $103(41 \%)$ & $77(50 \%)$ & & $149(45 \%)$ & $51(33 \%)$ & $47(27 \%)$ & \\
\hline \multicolumn{9}{|l|}{ Re-TURBT } \\
\hline No & $131(52 \%)$ & $83(50 \%)$ & $48(56 \%)$ & 0.3 & $111(54 \%)$ & $63(52 \%)$ & $48(56 \%)$ & 0.5 \\
\hline Yes & $120(48 \%)$ & $83(50 \%)$ & $37(44 \%)$ & & $94(46 \%)$ & $57(48 \%)$ & $37(44 \%)$ & \\
\hline
\end{tabular}

PSM propensity score matched, TURBT transurethral resection of bladder, IQR interquartile range, CIS carcinoma in situ, LVI lymphovascular invasion, $R e-T U R B T$ repeated TURBT 


\section{Cox regression analyses and survival estimates}

Median follow-up was 2.4 years (IQR 0.3-3.5). Table 2 resumes results of multivariable Cox regression analyses for recurrence, CSM and OM of the PSM cohort. Recurrence was associated with: pT2 vs. pT0/T1 stage [hazard ratio (HR) 2.01, CI 1.12-3.59, $p=0.02]$, pT3-T4 vs. pT0/T1 (HR 3.68, CI 2.03-6.67, $p<0.001$ ) and positive pathological nodal stage (HR 1.94, CI 1.25-3.04, $p=0.004$ ). CSM was found associated with pT3-T4 stages vs. pT0/T1 stage (HR 3.63 , CI 1.72-7.65, $p=0.001$ ), positive nodes (HR 3.00, CI $1.69-5.34, p<0.001)$ and number of lymph nodes removed (HR 0.95, CI 0.92-0.99, $p=0.01$ ), whereas OM was found associated with pT3-T4 stages vs. pT0/T1 stage (HR 1.82, CI 1.26-2.62, $p=0.001$ ), presence of CIS at RC specimens (HR 0.71, CI 0.53-0.95, $p=0.02$ ), presence of LVI (HR 0.53 , CI $0.37-0.74, p<0.001)$, presence of positive nodes
(HR 2.22, CI 1.57-3.14, $p<0.001$ ) and adjuvant chemotherapy (HR 0.36 , CI $0.20-0.64, p<0.001$ ). No significant association between extension of resection and recurrence $(p=0.2), \operatorname{CSM}(0=0.9)$ or OM $(p=0.8)$ was found at multivariable analyses. Kaplan-Meier curves depicting recurrence, CSM and OM are reported in Fig. 1.

\section{Prediction of adverse pathological features}

After applying the propensity score matching, 294 patients with c-TURBT were matched with 294 patients with incomplete TURBT. Table 3 reports results of multivariable logistic regression analyses predicting the relationship of c-TURBT on adverse pathological features of the PSM cohort. At multivariable analysis, associations between pT3-T4 stages and hydronephrosis [odds ratio (OR) 8.04, 95\% confidence interval (CI) 2.33-27.67, $p=0.001]$,
Table 2 Multivariable Cox regression analyses predicting the risk of recurrence, cancer-specific mortality (CSM) and overall mortality $(\mathrm{OM})$ in patients treated with radical cystectomy $(\mathrm{RC})$ and pelvic lymph node dissection (PLND) for very high risk non-muscle- invasive bladder cancer (BCa) or muscle-invasive $\mathrm{BCa}$ of propensity score match (PSM) cohort adjusted for all the variables included in the table

\begin{tabular}{|c|c|c|c|c|c|c|}
\hline \multirow[t]{2}{*}{ Variables } & \multicolumn{2}{|c|}{ Multivariable recurrence } & \multicolumn{2}{|c|}{ Multivariable CSM } & \multicolumn{2}{|l|}{ Multivariable OM } \\
\hline & HR $(95 \%$ CI $)$ & $p$ value & HR $(95 \% \mathrm{CI})$ & $p$ value & HR $(95 \% \mathrm{CI})$ & $p$ value \\
\hline Age & $0.98(0.96-1.00)$ & 0.2 & $0.98(0.95-1.01)$ & 0.2 & $1.00(0.98-1.01)$ & 0.9 \\
\hline Incomplete TURBT & $0.77(0.52-1.14)$ & 0.2 & $0.98(0.57-1.64)$ & 0.9 & $0.96(0.72-1.28)$ & 0.8 \\
\hline pT2 vs. pT0/T1 & $2.01(1.12-3.59)$ & 0.02 & $1.40(0.62-3.15)$ & 0.4 & $1.12(0.77-1.62)$ & 0.5 \\
\hline pT3-4 vs. pT0/T1 & $3.68(2.03-6.67)$ & $<0.001$ & $3.63(1.72-7.65)$ & 0.001 & $1.82(1.26-2.62)$ & 0.001 \\
\hline CIS at RC & $0.77(0.51-1.15)$ & 0.2 & $0.90(0.53-1.51)$ & 0.7 & $0.71(0.53-0.95)$ & 0.02 \\
\hline $\mathrm{LVI}$ at $\mathrm{RC}$ & $0.86(0.56-1.32)$ & 0.5 & $0.82(0.53-1.51)$ & 0.7 & $0.53(0.37-0.74)$ & $<0.001$ \\
\hline Variant histology at RC & $1.05(0.67-1.65)$ & 0.8 & $1.59(0.92-2.75)$ & 0.09 & $1.32(0.96-1.83)$ & 0.09 \\
\hline $\mathrm{pN}+$ & $1.94(1.25-3.04)$ & 0.004 & $3.00(1.69-5.34)$ & $<0.001$ & $2.22(1.57-3.14)$ & $<0.001$ \\
\hline lymph nodes removed, number & $0.99(0.97-1.02)$ & 0.9 & $0.95(0.92-0.99)$ & 0.01 & $1.01(0.99-1.02)$ & 0.2 \\
\hline Positive STSM & $0.93(0.49-1.77)$ & 0.9 & $0.40(0.14-1.14)$ & 0.09 & $0.60(0.35-1.03)$ & 0.06 \\
\hline Adjuvant chemotherapy & $1.58(0.90-2.77)$ & 0.1 & $0.80(0.34-1.91)$ & 0.6 & $0.36(0.20-0.64)$ & $<0.001$ \\
\hline
\end{tabular}

$C S M$ cancer-specific mortality, $O M$ overall mortality, $H R$ hazard ratio, $T U R B T$ transurethral resection of bladder, $C I S$ carcinoma in situ, $R C$ radical cystectomy, LVI lymphovascular invasion, LN lymph nodes, STSM soft tissue surgical margin
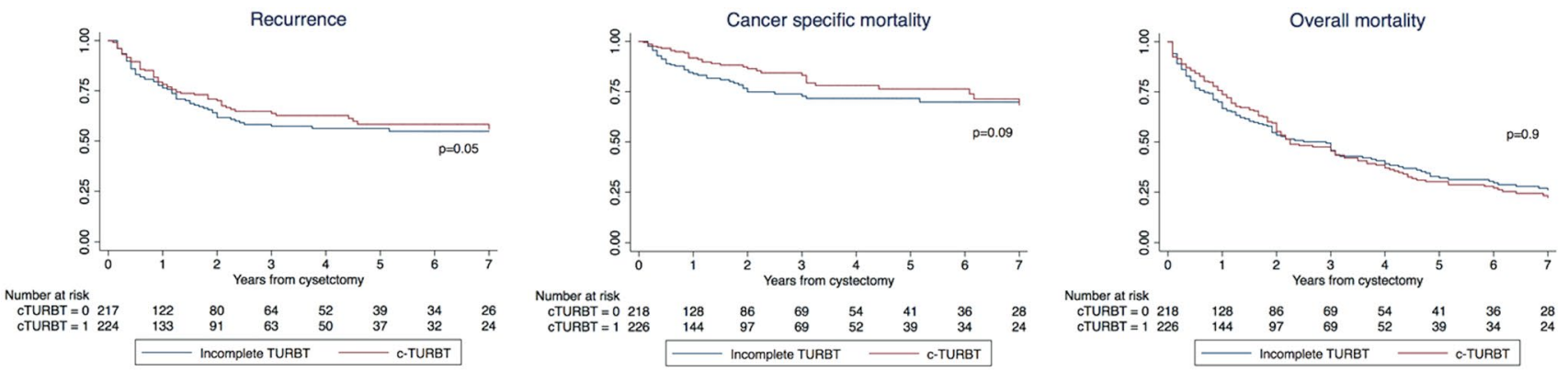

Fig. 1 Kaplan-Meier analyses assessing, recurrence, cancer-specific mortality (CSM) and overall mortality (OM) in patient treated with radical cystectomy (RC) and pelvic lymph node dissection (PLND) with previous complete and incomplete transurethral resection (TURBT) 
Table 3 Multivariable logistic regression analyses predicting pT3-T4 stage, positive soft tissue surgical margin (STSM) and lymph node invasion (LNI) at radical cystectomy (RC) of propensity score match (PSM) cohort

\begin{tabular}{|c|c|c|c|c|c|c|}
\hline \multirow[t]{3}{*}{ Variables } & \multicolumn{6}{|c|}{ Multivariable analyses } \\
\hline & \multicolumn{2}{|l|}{ pT3-T4 stage } & \multicolumn{2}{|l|}{ Positive STSM } & \multicolumn{2}{|l|}{ LNI } \\
\hline & OR $(95 \% \mathrm{CI})$ & $p$ value & OR $(95 \% \mathrm{CI})$ & $p$ value & OR $(95 \% \mathrm{CI})$ & $p$ value \\
\hline Age & $1.02(0.96-1.09)$ & 0.4 & $0.95(0.87-1.04)$ & 0.3 & $0.98(0.92-1.04)$ & 0.6 \\
\hline Preoperative hydronephrosis yes vs. no & $8.04(2.33-27.67)$ & 0.001 & $3.24(0.77-13.69)$ & 0.1 & $2.49(0.89-6.94)$ & 0.08 \\
\hline Clinical T3-T4 vs. clinical T1-T2 & $1.22(0.49-3.00)$ & 0.7 & $0.40(0.08-1.82)$ & 0.2 & $0.88(0.34-2.27)$ & 0.8 \\
\hline Concomitant CIS at TURBT yes vs. no & $0.45(0.10-1.99)$ & 0.3 & $1.46(0.13-16.08)$ & 0.8 & $0.93(0.16-5.40)$ & 0.9 \\
\hline Variants at TURBT yes vs. no & $11.4(1.98-65.94)$ & 0.06 & $0.84(0.08-8.15)$ & 0.9 & $0.11(0.01-0.97)$ & 0.06 \\
\hline Multifocality at TURBT yes vs. no & $0.34(0.13-0.87)$ & 0.02 & $1.84(0.43-7.77)$ & 0.4 & $0.91(0.33-2.49)$ & 0.9 \\
\hline TURBT stage $\geq 2$ vs. TURBT stage $<2$ & $4.06(1.43-11.46)$ & 0.002 & $1.95(0.36-10.60)$ & 0.4 & $2.43(0.68-8.66)$ & 0.2 \\
\hline Dimension $>3 \mathrm{~cm}$ at TURBT yes vs. no & $0.54(0.20-1.41)$ & 0.3 & $0.37(0.07-1.78)$ & 0.2 & $0.86(0.30-2.44)$ & 0.9 \\
\hline Incomplete TURBT yes vs. no & $2.78(1.11-7.00)$ & 0.03 & $1.46(0.35-6.10)$ & 0.6 & $0.92(0.25-2.40)$ & 0.9 \\
\hline Clinical $\mathrm{N}+$ yes vs. no & $1.08(0.38-3.01)$ & 0.9 & $4.48(0.89-22.43)$ & 0.07 & $1.02(0.34-3.04)$ & 0.9 \\
\hline
\end{tabular}

STSM soft tissue surgical margin, LNI lymph node invasion, OR odds ratio, 95\% CI confidence interval, CIS carcinoma in situ, TURBT transurethral resection

presence of multifocality (OR 0.34 , CI $0.13-0.83, p=0.02$ ) pathological T stage at TURBT specimens' $\geq 2$ (OR 4.06, CI $1.43-11.4, p=0.002$ ), and incompleteness of TURBT (OR 2.78, CI 1.11-7.00, $p=0.03$ ) were found, whereas no significant association was found at multivariable analysis with STSM and LNI.

\section{Discussion}

Although several studies reported a survival benefit in completing TURBT in non-muscle-invasive BCa treated with a conservative approach [3, 4, 12], scarce data exist regarding its role in patients candidate for RC. Specifically, it is not clear whether an extended resection is needed to improve oncological outcomes after RC. Understanding this aspect would be very useful for surgeons who are often led to interrupt the procedure for the fear of bladder perforation and subsequent tumor spreading. Therefore, bladder perforation represents a frequent complication during TURBT [13] and it seems to be associated with worse recurrence-free survival, $\mathrm{T}$ stage progression and consequent higher number of cystectomy [14]. Several risk factors are related to higher possibility to cause a bladder perforation, such as gender, body mass index [15], size of the tumor, number of tumors and their site [16], and these characteristics may lead to the decision to interrupt prematurely the procedure, leaving residual macroscopic cancer in the bladder.

In our study, we evaluated a large cohort of 727 patients treated in three tertiary referral centers. Of these patients, $433(60 \%)$ received a c-TURBT. Our results are in concordance with the previous literature: Adiyat et al. [17] reported a $30 \%$ rate of macroscopic residual disease in the site of the previous resection in non-muscle-invasive $\mathrm{BCa}$, whereas percentage increases until 50\% in muscle-invasive bladder cancer with a pT2 final stage [18]. The high rate of incompleteness of TURBT reported in the previous literature is probably dependent on several factors: first, especially as regards multifocal non-muscle-invasive $\mathrm{BCa}$, on the inadequacy of surgical techniques as the lack of narrow-band imaging (NBI) or photodynamic diagnosis (PDD) which can improve detection of tumors and consequently their resection $[19,20]$. Second, the experience of the surgeon: as reported by Mariappan et al. [3] experience is an independent factor of prediction of TURBT's quality because it can increase the rates of the presence of muscular tissue at the final pathological evaluation, can enhance resection of tumors located on the anterior wall, dome and diverticula and finally can improve "en bloc" resection for minor tumors and complete fractioned resection of tumors with greater dimension. Third, the need of limited anesthesiologic time due to patient's comorbidities or necessity of contained time in order to avoid, even its rarity, TURBT syndrome related to bladder resection [21]. In our study, all the surgeons were experts in endoscopic surgery, whereby the main cause of the high rate of incompleteness of TURBT can be imputed to the occurrence of complication and to the lack of use of PDD and NBI before their introduction: Both these techniques are currently suggested and performed according to guidelines, but they have been introduced later compared to the beginning of our study. Unfortunately, no data regarding the possible cause of incompleteness of TURBT were reported in our database.

Moreover, in our study, $3.0 \%$ only of patients who underwent a c-TURBT reached a final pT0-pTa-pTis stage at RC. If we add to this value the percentage of 
pT1 residual disease at RC pathological evaluation, the overall rate increases to $47 \%$ in the reported c-TURBT. These findings support the subjectivity of evaluation of completeness of TURBT and validate the probable high incidence of microscopic residual cancers after endoscopic resection [22]. However, the proportion of patients who achieved a pT0-pTa-pTis stage at the time of cystectomy increased from $2.0 \%$ in patients with macroscopic incomplete TURBT to $3.0 \%$ in c-TURBT. Our finding supports Thrasher et al. [18] study, which reported a higher percentage of achievement a pT0 stage at RC specimen in patients with a reported macroscopic complete resection.

In our study, at PSM multivariable analyses, incompleteness of TURBT was an independent predictor of adverse pathological stage at RC specimens $(p=0.03)$. This result is probably related to a greater difficulty in the endoscopic management of more locally advanced tumors, especially for extravesical diseases. Supporting this, in our study incompleteness of TURBT was also associated with the presence of preoperative hydronephrosis, which is often an indirect signal of the presence of muscleinvasive bladder cancer and more advanced pathological stage in cystectomy series $[23,24]$. No relationship was found between c-TURBT and oncological outcomes. On the contrary, adverse pathological stage (pT3-pT4) was significantly associated with recurrence, CSM and OM: This result suggests that, after adjustment with all the confounders, the pathological $\mathrm{T}$ stage is a stronger predictor of survival outcomes compared to incompleteness of TURBT. This finding could be helpful especially for treating patients "unfit" for neoadjuvant chemotherapy as results of their comorbidities. Patients with high Charlson comorbidity index have frequently also a high American Society of Anesthesiologic (ASA) score. We believe that elderly patients unfit for NAC, who have resectable tumors which give impression of a muscle-invasive disease, can be treated with a stage resection, since endoscopic debulking of the tumor has not consequences on recurrence and survival after radical cystectomy.

Moreover, although international guidelines recommend NAC for cT2-4a BCa, Culp et al. [25] looking for a strategy for improving patients selection for neoadjuvant therapy found that patients with pT3-4 diseases are those who might benefit more from NAC, whereas patients with organ-confined diseases benefit less from this treatment. It should be considered, in addition to Culp finding, that several studies reported inadequacy of clinical staging techniques for bladder cancer with more than $50 \%$ of extravesical diseases being under-staged. The result we have found regarding the significant relationship between incompleteness of TURBT ad adverse pathological $\mathrm{T}$ stage can improve patients selection for NAC itself, driving the decision-making especially in doubtful cases.
This study is not without limitations. First of all, our study is limited by its retrospective design. Nevertheless, in the centers involved in this study, data were recorded prospectively and were transferred adequately to the databases. Second is the extended study period spanning almost 15 years, in which diagnostic techniques (example CT scan) and endoscopic tools (example introduction of NBI and PDD) were improved. Third is the lack of data regarding the use of NBI and PDD for each procedure, the cause of incompleteness of TURBT and time from TURBT itself and radical cystectomy. Fourth, TURBT was performed by different surgeons, although all of them were expert in endoscopic procedures. Fifth, the dimension of our cohort and the lack of data regarding the presence of lymphovascular invasion at TURBT have limited the possibility to perform analyses by subgroups of patients. Lastly, our evaluation of recurrence-free survival includes any recurrences, whereas it would be probably advisable to perform a separate analysis for evaluation of c-TURBT just in the local ones, even if in several works the relationship between pathologic $\mathrm{T}$ stage and any kind of recurrence, included the distant ones, was demonstrated [26-28]. Despite these limitations, our analyses provided worthwhile information for the effectiveness of c-TURBT over adverse pathological features and survival outcomes in overall population who undergoes RC with curative intent.

\section{Conclusion}

More than half of our cohort received a reported c-TURBT. However, just a small proportion of these patients reached a final pT0 stage, confirming the high probability of microscopic residual disease after endoscopic resection. Incompleteness of TURBT was found significantly associated with adverse $\mathrm{pT}$ stage at RC specimens' but not with worse survival outcomes compared to patients who received a c-TURBT. Given the current inadequacy of clinical staging strategies in $\mathrm{BCa}$ with more than $50 \%$ of extravesical disease being under-staged, our results could improve patients selection for NAC, driving the decision-making in doubtful cases. Given the scarcity of data from the published literature, a prospective randomized trial is needed to confirm our results.

Authors contribution SZ contributed to manuscript writing, statistical analyses and data collection. MM contributed to project development, data collection and drafting of the manuscript. AG contributed to critical revision of the manuscript. RC contributed to critical revision of the manuscript. FM contributed to critical revision of the manuscript. $\mathrm{AB}$ contributed to critical revision of the manuscript. AS contributed to critical revision of the manuscript. AA contributed to critical revision of the manuscript. CS contributed to critical revision of the manuscript. 
SB contributed to drafting of the manuscript. LC contributed to drafting of the manuscript. AM contributed to critical revision of the manuscript. PB contributed to project development, supervision and critical revision of the manuscript.

\section{Compliance with ethical standards}

Conflict of interest The authors declare that they have no conflict of interest.

Informed consent All persons gave their informed consent to use their data for this retrospective study.

Ethical approval All procedures performed in studies involving human participants were in accordance with the ethical standards of the institutional and/or national research committee and with the 1964 Helsinki Declaration and its later amendments or comparable ethical standards.

\section{References}

1. Siegel RL, Miller KD, Jemal A (2018) Cancer statistics, 2018. CA Cancer J Clin 68:7-30. https://doi.org/10.3322/caac.21442

2. Babjuk M, Böhle A, Burger M, Capoun O, Cohen D, Compérat EM et al (2017) EAU guidelines on non-muscle-invasive urothelial carcinoma of the bladder: update 2016. Eur Urol 71:447-461. https://doi.org/10.1016/j.eururo.2016.05.041

3. Mariappan P, Finney SM, Head E, Somani BK, Zachou A, Smith $G$ et al (2012) Good quality white-light transurethral resection of bladder tumours (GQ-WLTURBT) with experienced surgeons performing complete resections and obtaining detrusor muscle reduces early recurrence in new non-muscle-invasive bladder cancer: validation across time and place and recommendation for benchmarking. BJU Int 109:1666-1673. https://doi.org/10.1111/ j.1464-410X.2011.10571.x

4. Divrik RT, Sahin AF, Yildirim U, Altok M, Zorlu F (2010) Impact of routine second transurethral resection on the long-term outcome of patients with newly diagnosed pT1 urothelial carcinoma with respect to recurrence, progression rate, and disease-specific survival: a prospective randomised clinical trial. Eur Urol 58:185190. https://doi.org/10.1016/j.eururo.2010.03.007

5. Chang SS, Bochner BH, Chou R, Dreicer R, Kamat AM, Lerner SP et al (2017) Treatment of non-metastatic muscle-invasive bladder cancer: AUA/ASCO/ASTRO/SUO guideline. J Urol 198:552559. https://doi.org/10.1016/j.juro.2017.04.086

6. Alfred Witjes J, Lebret T, Compérat EM, Cowan NC, De Santis M, Bruins HM et al (2017) Updated 2016 EAU guidelines on muscle-invasive and metastatic bladder cancer. Eur Urol 71:462475. https://doi.org/10.1016/j.eururo.2016.06.020

7. Mostofi FK, Sobin LH, Torloni H, World Health Organization (1973) Histological typing of urinary bladder tumours. In: Mostofi FK (ed) Collaboration with Sobin LH, Torloni H, and pathologists in fourteen countries. World Health Organization, Geneva

8. Montironi R, Lopez-Beltran A (2005) The 2004 WHO classification of bladder tumors: a summary and commentary. Int J Surg Pathol 13:143-153. https://doi.org/10.1177/106689690501300203

9. Shariat SF, Svatek RS, Tilki D, Skinner E, Karakiewicz PI, Capitanio $U$ et al (2010) International validation of the prognostic value of lymphovascular invasion in patients treated with radical cystectomy. BJU Int 105:1402-1412. https://doi.org/10.1111/ j.1464-410X.2010.09217.x

10. Moschini M, D’Andrea D, Korn S, Irmak Y, Soria F, Compérat E et al (2017) Characteristics and clinical significance of histological variants of bladder cancer. Nat Rev Urol 14:651668. https://doi.org/10.1038/nrurol.2017.125

11. AJCC Cancer Staging Manual. In: Greene FL (eds). Springer (2018). https://www.springer.com/us/book/9781475736564. Accessed 3 Oct 2018

12. Brausi M, Collette L, Kurth K, van der Meijden AP, Oosterlinck W, Witjes JA et al (2002) Variability in the recurrence rate at first follow-up cystoscopy after TUR in stage Ta T1 transitional cell carcinoma of the bladder: a combined analysis of seven EORTC studies. Eur Urol 41:523-531

13. Avallone MA, Sack BS, El-Arabi A, Charles DK, Herre WR, Radtke AC et al (2017) Ten-year review of perioperative complications after transurethral resection of bladder tumors: analysis of monopolar and plasmakinetic bipolar cases. J Endourol 31:767-773. https://doi.org/10.1089/end.2017.0056

14. Comploj E, Dechet CB, Mian M, Trenti E, Palermo S, Lodde M et al (2014) Perforation during TUR of bladder tumours influences the natural history of superficial bladder cancer. World J Urol 32:1219-1223. https://doi.org/10.1007/s00345-013-1197-x

15. Herkommer K, Hofer C, Gschwend JE, Kron M, Treiber U (2012) Gender and body mass index as risk factors for bladder perforation during primary transurethral resection of bladder tumors. J Urol 187:1566-1570. https://doi.org/10.1016/j. juro.2011.12.114

16. Balbay MD, Cimentepe E, Unsal A, Bayrak O, Koç A, Akbulut $\mathrm{Z}$ (2005) The actual incidence of bladder perforation following transurethral bladder surgery. J Urol 174:2260-2262. https://doi. org/10.1097/01.ju.0000181811.61199.35 (discussion 2262-2263)

17. Adiyat KT, Katkoori D, Soloway CT, De los Santos R, Manoharan M, Soloway MS (2010) "Complete transurethral resection of bladder tumor": are the guidelines being followed? Urology 75:365-367. https://doi.org/10.1016/j.urology.2009.08.082

18. Lee SE, Jeong IG, Ku JH, Kwak C, Lee E, Jeong JS (2004) Impact of transurethral resection of bladder tumor: analysis of cystectomy specimens to evaluate for residual tumor. Urology 63:873-877. https://doi.org/10.1016/j.urology.2003.12.035 (discussion 877)

19. Mowatt G, N'Dow J, Vale L, Nabi G, Boachie C, Cook JA et al (2011) Photodynamic diagnosis of bladder cancer compared with white light cystoscopy: systematic review and meta-analysis. Int J Technol Assess Health Care 27:3-10. https://doi.org/10.1017/ S0266462310001364

20. Cauberg ECC, Kloen S, Visser M, de la Rosette JJMCH, Babjuk M, Soukup V et al (2010) Narrow band imaging cystoscopy improves the detection of non-muscle-invasive bladder cancer. Urology 76:658-663. https://doi.org/10.1016/j.urolo gy.2009.11.075

21. Hahn RG (1995) Transurethral resection syndrome after transurethral resection of bladder tumours. Can J Anaesth 42:69-72. https ://doi.org/10.1007/BF03010574

22. Herr HW (1999) The value of a second transurethral resection in evaluating patients with bladder tumors. J Urol 162:74-76. https ://doi.org/10.1097/00005392-199907000-00018

23. Haleblian GE, Skinner EC, Dickinson MG, Lieskovsky G, Boyd SD, Skinner DG (1998) Hydronephrosis as a prognostic indicator in bladder cancer patients. J Urol 160:2011-2014

24. Bartsch GC, Kuefer R, Gschwend JE, de Petriconi R, Hautmann RE, Volkmer BG (2007) Hydronephrosis as a prognostic marker in bladder cancer in a cystectomy-only series. Eur Urol 51:690 697. https://doi.org/10.1016/j.eururo.2006.07.009 (discussion 697-698)

25. Culp SH, Dickstein RJ, Grossman HB, Pretzsch SM, Porten S, Daneshmand $S$ et al (2014) Refining patient selection for neoadjuvant chemotherapy before radical cystectomy. J Urol 191:40-47. https://doi.org/10.1016/j.juro.2013.07.061

26. Stein JP, Lieskovsky G, Cote R, Groshen S, Feng AC, Boyd S et al (2001) Radical cystectomy in the treatment of invasive bladder 
cancer: long-term results in 1054 patients. J Clin Oncol 19:666675. https://doi.org/10.1200/JCO.2001.19.3.666

27. Bruins HM, Huang GJ, Cai J, Skinner DG, Stein JP, Penson DF (2009) Clinical outcomes and recurrence predictors of lymph node positive urothelial cancer after cystectomy. J Urol 182:2182-2187. https://doi.org/10.1016/j.juro.2009.07.017

28. Umbreit EC, Crispen PL, Shimko MS, Farmer SA, Blute ML, Frank I (2010) Multifactorial, site-specific recurrence model after radical cystectomy for urothelial carcinoma. Cancer 116:33993407. https://doi.org/10.1002/cncr.25202

Publisher's Note Springer Nature remains neutral with regard to jurisdictional claims in published maps and institutional affiliations. 